\title{
Assessment and Management of Acute Disseminated Encephalomyelitis (ADEM) in the Pediatric Patient
}

\author{
Cynthia X. Wang ${ }^{1,2}$ (1)
}

Accepted: 8 March 2021 / Published online: 8 April 2021

(c) This is a U.S. government work and not under copyright protection in the U.S.; foreign copyright protection may apply 2021

\begin{abstract}
Acute disseminated encephalomyelitis (ADEM) is an inflammatory demyelinating disease of the central nervous system that typically presents in childhood and is associated with encephalopathy and multifocal brain lesions. Although ADEM is thought to be a post-infectious disorder, the etiology is still poorly understood. ADEM is often a monophasic disorder, in contrast to other demyelinating disorders such as multiple sclerosis and neuromyelitis optica spectrum disorder. With increasing awareness, understanding, and testing for myelin oligodendrocyte glycoprotein antibodies, this disease is now known to be a cause of pediatric ADEM and also has the potential to be relapsing. Diagnostic evaluation for ADEM involves neuroimaging and laboratory studies to exclude potential infectious, inflammatory, neoplastic, and genetic mimics of ADEM. Acute treatment modalities include high-dose intravenous corticosteroids, therapeutic plasma exchange, and intravenous immunoglobulin. Long-term outcomes for ADEM are generally favorable, but some children have significant morbidity related to the severity of acute illness and/or manifest ongoing neurocognitive sequelae. Further research related to the optimal management of pediatric ADEM and its impact on prognosis is needed. This review summarizes the current knowledge of the pathogenesis, epidemiology, clinical features, diagnostic evaluation, treatment approaches, and outcomes in pediatric ADEM.
\end{abstract}

\section{Introduction}

Acute disseminated encephalomyelitis (ADEM) is an inflammatory demyelinating disease of the central nervous system (CNS) that typically presents with encephalopathy and multifocal brain lesions. This condition more commonly affects children and is often a monophasic illness with good functional recovery. Diagnostic testing should be aimed at differentiating ADEM from potential infectious, toxic/metabolic, genetic, vascular, and neoplastic mimics. It is also important to consider whether ADEM represents the first attack of a relapsing inflammatory demyelinating syndrome, particularly myelin oligodendrocyte glycoprotein (MOG) antibody disease or, less likely,

Cynthia X. Wang

cynthia.wang@utsouthwestern.edu

1 Department of Neurology and Neurotherapeutics, University of Texas Southwestern Medical School, 5323 Harry Hines Blvd, Dallas, TX 75390, USA

2 Department of Pediatrics, University of Texas Southwestern Medical School, 5323 Harry Hines Blvd, Dallas, TX 75390, USA

\section{Key Points}

Acute disseminated encephalomyelitis can have many different presenting features, but the hallmark is multifocal neurological deficits associated with encephalopathy. Diagnostic workup involves serum and spinal fluid studies to investigate infectious and inflammatory causes and neuroimaging to characterize the extent of central nervous system involvement.

Acute therapies such as high-dose intravenous corticosteroids, therapeutic plasma exchange, and intravenous immunoglobulin are aimed at reducing inflammation and generally lead to marked clinical improvement.

Long-term follow-up of pediatric patients is essential to mitigate any potential neurological or psychosocial sequelae of the condition. 
multiple sclerosis or neuromyelitis optica spectrum disorder (NMOSD).

Once testing is sufficiently suggestive of an acute demyelinating syndrome, it is critical that treatments directed at reducing inflammation and immune activation are started to decrease the duration and severity of the illness. Early initiation of physical, occupational, and speech therapy, when applicable, can help facilitate earlier and more complete recovery. While long-term prognosis and physical functioning is good, increasing data suggest that children with ADEM can have persistent neurocognitive deficits. A multidisciplinary approach to the follow-up of a child with ADEM, involving the expertise of neurologists, neuropsychologists, and physiatrists, among other clinicians, is important to optimize disease recovery and facilitate surveillance for potential relapses of acute demyelination.

\section{Pathogenesis}

ADEM is postulated to be an autoimmune disorder in which an environmental stimulus triggers a dysfunctional, exaggerated immune response in genetically susceptible individuals [1]. ADEM has also been referred to as postinfectious encephalomyelitis based on this suspected mechanism of a pathogen triggering the aberrant immune response. It is thought that myelin constituents such as myelin basic protein, MOG, and myelin proteolipid protein share antigenic determinants with that of an inciting pathogen in a process termed molecular mimicry $[2,3]$.

Many pathogens have been reported to have an association with ADEM, including measles, rubella, varicella zoster, influenza, Epstein-Barr virus, herpes simplex virus (HSV), enterovirus, coxsackievirus, mycoplasma pneumonia, borrelia burgdorferi, and beta-hemolytic Streptococcus [4]. In 2020, the global pandemic associated with severe acute respiratory syndrome coronavirus 2 (SARS-CoV-2) infection resulted in multiple reports of associated ADEM [5, 6]. Given that numerous infectious organisms can trigger ADEM, alternative mechanisms of pathogenesis may include activation of existing autoreactive lymphocytes through a nonspecific inflammatory process and entry into the CNS by transient breakdown of the blood-brain barrier [7].

Prior vaccines that were contaminated with neural tissue, including the Semple rabies vaccine, led to an increased incidence of ADEM [8]. Currently, most evidence suggests no significant association between ADEM and prior immunization [9]. Most historical data, including the incidence of measles following natural infection versus immunization, suggests that the risk of ADEM is many folds higher in the former $[10,11]$.

\section{Epidemiology}

ADEM is a rare illness with an incidence of $0.2-0.4$ per 100,000 children annually $[12,13]$. The most common age of presentation is between 3 and 7 years [14]. There is a slight male predominance but no specific ethnic predilection [15]. In up to $75 \%$ of ADEM cases, a febrile upper respiratory or gastrointestinal illness precedes the onset of neurological symptoms [16, 17]. Some studies have reported a seasonal predilection for winter and spring [18].

\section{Clinical Features}

In the typical presentation of ADEM, neurological symptoms develop 1-2 weeks following an infection and may involve headache, emesis, meningismus, and alterations in behavior and level of alertness. Encephalopathy and multifocal neurological deficits are the cardinal symptoms of ADEM. The degree of encephalopathy can vary greatly and may include irritability, confusion, somnolence, lethargy, and stupor. The usual progression from onset to maximal severity of symptoms occurs over 4-7 days. Common exam findings include altered mental status, ataxia, and extremity weakness. ADEM can also be associated with optic neuritis and transverse myelitis, leading to vision changes and extremity weakness/urinary retention, respectively.

The acute changes associated with ADEM usually last 2-4 weeks, during which time children may require hospitalization for medical stabilization, diagnostic workup, treatment, and rehabilitation. In severe cases, children may require intensive care unit stays for respiratory insufficiency, refractory seizures, and severe encephalopathy. Some conditions thought to be variants of ADEM can be fatal, including acute hemorrhagic leukoencephalitis (also known as Weston-Hurst syndrome) and acute necrotizing encephalitis of childhood [19]. These have been associated with specific inciting infections and genetic mutations [20].

\section{Diagnostic Evaluation}

ADEM is primarily a clinical and radiological diagnosis, with no specific confirmatory laboratory tests or pathognomonic imaging findings. It rests on excluding alternate etiologies, particularly infections and other inflammatory demyelinating syndromes. The International Pediatric Multiple Sclerosis Study Group defined ADEM as follows [21]:

- A first polyfocal, clinical CNS event with presumed inflammatory demyelinating cause.

- Encephalopathy that cannot be explained by fever. 
- No new clinical and magnetic resonance imaging (MRI) findings emerge $\geq 3$ months after the onset.

- Brain MRI is abnormal during the acute (3-month) phase.

The following sections outline the studies that should be obtained when ADEM is suspected in a child who presents with multifocal neurological deficits.

\subsection{Neuroimaging}

\subsubsection{Magnetic Resonance Imaging (MRI) Brain}

MRI brain with and without contrast can be used to evaluate for the multifocal brain lesions characteristic of ADEM. T2-weighted fluid-attenuated inversion recovery (T2-FLAIR) sequences often demonstrate the pathology best, which typically appears as poorly demarcated and asymmetric hyperintensities, ranging in size from $<1 \mathrm{~cm}$ to multiple-centimeter confluent white matter abnormalities. Typically, lesions occur in the deep and subcortical white matter while sparing periventricular white matter, unlike multiple sclerosis. Hypointense lesions on T1-weighted images ("black holes") are less common in ADEM and are more suggestive of multiple sclerosis, as they indicate prior demyelination. T1-weighted images with gadolinium contrast can identify areas of enhancement, indicating active inflammation and loss of blood-brain barrier integrity. Gray matter lesions can occur in ADEM, particularly in the thalami and basal ganglia. Hemorrhagic and hyperacute variants of ADEM may show areas of acute hemorrhage, visualized best on susceptibility-weighted MRI sequences, and lesions that restrict diffusion of water on diffusion-weighted sequences.

\subsubsection{MRI Spine}

MRI spine with and without contrast should be considered if a child demonstrates sensory changes, particularly a sensory level, extremity weakness, and/or bowel and bladder dysfunction. Abnormal hyperintense signal on T2 and short tau inversion recovery (STIR) images can involve the central gray matter, peripheral white matter, or a mixed pattern. Spinal cord lesions can be short, spanning one to two vertebral segments, or longitudinally extensive, extending to three or more vertebral segments. In adults, longitudinally extensive transverse myelitis (LETM) is highly associated with aquaporin-4 NMOSD, but longer lesions are more common in children. Short, wedge-shaped, eccentric lesions are more frequently seen in multiple sclerosis. Anti-MOG-related myelitis can present with LETM, and these lesions may have a gray matter-predominant pattern, sometimes making it difficult to differentiate from acute flaccid myelitis [22]. Anti-MOG disease also tends to affect lower segments of the spinal cord, such as the lower thoracic and lumbar cord, including conus medullaris, which is less common in multiple sclerosis and NMOSD [23].

\subsection{Cerebrospinal Fluid Studies}

Spinal fluid studies are critical for investigating infectious and inflammatory etiologies for CNS inflammation. Cerebrospinal fluid (CSF) analysis should include cell count and differential, protein, glucose, and opening pressure. Based on level of suspicion for infection, studies such as CSF culture, viral studies (HSV-1 and -2, enterovirus, influenza, Epstein-Barr virus, varicella, West Nile virus, cytomegalovirus polymerase chain reaction), and bacterial/viral serologies (Borrelia, syphilis, Mycoplasma, rubella) can be obtained, particularly if there are concerning signs such as fever, meningismus, and meningeal enhancement.

CSF pleocytosis and increased protein are common in patients with ADEM. Red blood cells may be increased in hemorrhagic variants of ADEM but should also raise concern for infectious alternatives such as HSV encephalitis. Opening pressure may be elevated and should prompt close continued monitoring for signs of increased intracranial pressure.

Assessment of intrathecal antibody synthesis through oligoclonal bands and immunoglobulin $\mathrm{G}(\mathrm{IgG})$ index can be informative. The presence of two or more unique oligoclonal bands in CSF can be seen in multiple sclerosis, though alternative etiologies such as CNS infections and anti-N-methylD-aspartate encephalitis could produce similar findings. Fewer than $20 \%$ of children with ADEM have oligoclonal bands in the CSF [24]. Neuromyelitis optica (NMO) IgG can also be assessed in CSF, but serum may be more sensitive. MOG IgG testing in CSF could also be considered but is not widely commercially available.

\subsection{Serum Studies}

Testing for MOG IgG and NMO IgG are increasingly utilized, as these cell-based assays are both sensitive and specific for the respective CNS demyelinating disorders. It is important to assess for these conditions as they require closer follow-up given their potential to be relapsing syndromes, particularly NMOSD, in which immunosuppressive therapy should be initiated after the incident attack. NMO IgG is a very specific biomarker for an NMOSD. Typically, these patients have symptoms of optic neuritis or transverse myelitis, though brain and brainstem lesions can be seen, particularly in the dorsal medulla, that can be clinically associated with intractable hiccups and vomiting (area postrema syndrome). If untreated, individuals with this condition have 
a high likelihood of further attacks that lead to significant visual and motor disability. Increasing data suggest that individuals with persistent seropositivity to MOG IgG are also at risk for relapses, whereas those who become seronegative are more likely to have a monophasic illness [25].

\subsection{Adjunctive Tests}

Consider additional testing based on any elements of the history that raise concern for alternate diagnoses such as infectious, neoplastic, vascular, or metabolic/genetic/mitochondrial disorders.

Elevated inflammatory markers such as C-reactive protein and erythrocyte sedimentation rate can support acute inflammation/infection. If infection is considered, viral and bacterial serologies can be obtained to evaluate for a parainfectious or postinfectious syndrome. Next-generation metagenomic sequencing has been increasingly utilized to diagnose less common infections of the CNS [26].

Vascular disorders typically have a hyperacute presentation, with symptom onset to peak severity emerging over minutes to hours. In contrast, metabolic and genetic conditions have an indolent progression, spanning months or years. Compared with adults, children have a relatively greater number of alternate diagnoses for ADEM, such as genetic disorders and inborn errors of metabolism. These should be considered in children who have a history of developmental delay, elevations of serum or CSF lactate, and poor responsiveness to standard treatment.

In children with persistent changes in mental status or poor level of alertness, an electroencephalogram can be helpful to characterize the degree of encephalopathy as defined by focal or generalized slowing of the brain waves and to investigate for subclinical seizures that could be contributing to altered mentation.

In very rare cases, a brain biopsy may be indicated, particularly if primary angiitis of the CNS is considered as part of the differential diagnosis. Alternatively, large tumefactive lesions may raise concern for a neoplastic process. If so, CSF evaluation for cytology and flow cytometry can also be considered. Hemophagocytic lymphohistiocytosis workup should be considered in a child who has symptoms of fever, elevated ferritin, elevated liver function tests, and cytopenias.

\section{Approach to Treatment of Acute Disseminated Encephalomyelitis}

While performing the initial workup for ADEM, empirical antimicrobials should be considered in a child presenting with fever, meningismus, acute encephalopathy, and evidence of inflammation in the spinal fluid and/or MRI brain. Hypoglycorrhachia, defined as glucose $<45 \mathrm{mg} / \mathrm{dL}$ or CSFto-serum glucose ratio $\leq 0.5$, strongly suggests an infectious etiology.

If ADEM is felt to be the most likely diagnosis, acute therapies such as high-dose corticosteroids, intravenous immunoglobulin (IVIG), and therapeutic plasma exchange (PLEX) are commonly employed. Immunotherapy may be used concurrently with antibiotics and antivirals until the results of these studies return.

No randomized controlled trials have been completed to support practice guidelines for acute management of ADEM. The rationale for using immunotherapies for ADEM is primarily supported by results from small observational studies and expert consensus [27]. Table 1 summarizes the acute treatments for ADEM.

\subsection{First-Line Therapy: High-Dose Intravenous Corticosteroids}

Intravenous corticosteroids such as methylprednisolone are commonly used in children with suspected ADEM [28, 29]. This is commonly dosed at $30 \mathrm{mg} / \mathrm{kg}$ daily (up to $1000 \mathrm{mg}$ ) for 3-5 days. Alternative regimens include methylprednisolone at lower doses such as $10 \mathrm{mg} / \mathrm{kg}$ or dexamethasone $1-2 \mathrm{mg} / \mathrm{kg} /$ day for 3-5 days. The mechanisms of action of high-dose corticosteroids include dampening the inflammatory cytokine cascade, inhibiting the activation of $\mathrm{T}$ cells,

Table 1 Acute treatments for acute disseminated encephalomyelitis (ADEM)

\begin{tabular}{|c|c|c|c|}
\hline Line of treatment & Therapy & Dosing & Potential adverse effects \\
\hline First line & Intravenous methylprednisolone & $30 \mathrm{mg} / \mathrm{kg} /$ day for $3-5$ days & $\begin{array}{l}\text { Behavioral/mental status changes, insomnia, } \\
\text { hyperglycemia, hypertension, gastrointestinal } \\
\text { disturbances, risk of infections }\end{array}$ \\
\hline \multirow[t]{2}{*}{ Second line } & Therapeutic plasma exchange (PLEX) & $\begin{array}{l}1-1.5 \text { plasma volume exchanges } \\
\text { every other day for } 5-7 \text { treat- } \\
\text { ments }\end{array}$ & $\begin{array}{l}\text { Hypotension, hypocalcemia, pain, bleeding, } \\
\text { infection }\end{array}$ \\
\hline & Intravenous immunoglobulin (IVIG) & $2 \mathrm{~g} / \mathrm{kg}$ divided over $3-5$ days & $\begin{array}{l}\text { Thromboembolic events, acute kidney injury, } \\
\text { aseptic meningitis, headache, nausea, fever, } \\
\text { rash }\end{array}$ \\
\hline
\end{tabular}


decreasing the extravasation of immune cells into the CNS, and facilitating the apoptosis of activated immune cells [30, 31].

Following initiation of intravenous corticosteroids, a child's clinical status should be monitored closely to follow changes in mental status, strength, gait, coordination, sensation, and/or vision. Meaningful improvement may take several days to occur. Lack of improvement or deterioration may be a prognostically poor sign, suggesting either a fulminant variant of ADEM or potentially an alternative diagnosis.

If symptoms are significantly improved, no additional immunotherapy may be necessary. It is common practice to subsequently use a 4- to 6-week taper of an oral corticosteroid such as prednisone at $1-2 \mathrm{mg} / \mathrm{kg} /$ day (up to $60 \mathrm{mg}$ daily) initially, decreasing by $5-10 \mathrm{mg}$ weekly. The rationale for a steroid taper includes its potential role in reducing rebound disease activity from the acute ADEM episode and the possibility of immunomodulation that decreases the likelihood of future attacks of acute demyelination. Some observational studies have found that children who had corticosteroid tapers shorter than 3 weeks were more likely to have a relapse, although results were not statistically significant $[32,33]$.

In general, short-term use of high-dose corticosteroids is well-tolerated. Potential complications include behavioral and mental status changes, insomnia, hyperglycemia, hypertension, gastrointestinal disturbances, and increased susceptibility to infection.

\subsection{Second-Line Therapies}

Many children with ADEM respond favorably to high-dose corticosteroids alone and may not need additional immunotherapies. However, deficits such as inability to ambulate, severe and/or bilateral vision loss, persistent alteration in mental status, or refractory seizures may warrant the concurrent or sequential administration of IVIG and/or therapeutic PLEX.

At our center, our most commonly utilized second-line therapy is therapeutic PLEX. In our experience, this treatment is more efficacious than IVIG and allows for IVIG to be given following PLEX if needed, without decreasing the therapeutic effect of the former, which would not be true if IVIG were administered before PLEX.

\subsubsection{Therapeutic Plasma Exchange}

This therapy is typically administered as $1-1.5$ plasma volume exchanges every other day for five to seven treatments [34]. One retrospective cohort study of PLEX in children with CNS demyelination found the treatment was well-tolerated and associated with additional improvement following corticosteroids [35]. The mechanism of action involves the removal of circulating pathogenic immunoglobulins, immune complexes, complement, and cytokines. Typically, a significant clinical response becomes evident following the third or fourth PLEX. Potential complications and adverse events include hypotension, hypocalcemia, pain, bleeding, and infection related to central venous catheter placement. Albumin is the typical replacement fluid, but crystalloids can also be used.

\subsubsection{Intravenous Immunoglobulin}

IVIG is composed of pooled IgG from many donors. The mechanism of this treatment is hypothesized to involve decreasing the endogenous production of immunoglobulins, facilitating degradation of native immunoglobulins, and interfering with pathogenic immune complexes. IVIG has been utilized in pediatric patients with ADEM with refractory symptoms following corticosteroids with positive results [36-38]. Typically, it is dosed at $2 \mathrm{~g} / \mathrm{kg}$ divided over 3-5 days. Potential adverse events include hyperviscosity leading to thromboembolic events, acute kidney injury, aseptic meningitis, and infusion reactions of headache, nausea, fever, and rash. Anaphylactic reactions can occur in individuals with immunoglobulin $\mathrm{A}$ (IgA) deficiency, as most formulations of IVIG have some IgA contamination.

\subsection{Other Acute Management Considerations}

Hyperacute and fulminant variants of ADEM may lead to increased intracranial pressure [39, 40]. Medical management through hyperosmolar therapy to decrease cerebral edema and barbiturate medications to lower metabolic demands should be considered. When maximal medical therapy is not sufficient, neurosurgical consultation can be requested for evaluation for invasive interventions such as intracranial pressure monitoring, external ventricular drain, and hemicraniectomy in rare, life-threatening cases.

In cases of fulminant and refractory ADEM variants, therapies such as rituximab and/or cyclophosphamide have been utilized with some positive results [41-44]. However, the vast majority of cases of pediatric ADEM respond well to first- and second-line therapies, and lack of expected response should increase suspicion for alternative diagnoses and consideration of the impact of ADEM sequelae such as hemorrhage, increased intracranial pressure, and hypoxicischemic injury.

\subsection{Supportive Care and Therapies}

Close monitoring for airway protection must be continued in patients with altered mental status and escalated to mechanical ventilation if required. Other supportive measures 
include antiseizure medications, correction of fluid and electrolyte disturbances, and prophylactic anticoagulation for prevention of deep vein thrombosis in patients with high risk [45].

While a child is receiving immunotherapies, deficits in gross motor, fine motor, and speech/swallowing function can be assessed by inpatient therapists and rehabilitation physicians. Early initiation of physical, occupational, and speech therapy, when applicable, can help facilitate earlier and more complete recovery [46].

\subsection{Treatment Outcome}

In general, clinical symptoms and MRI lesions related to ADEM will regress over time following acute immunotherapy. By 3 months following the initial attack, most acute neurological deficits will have resolved, and associated brain lesions will demonstrate significant improvement or complete resolution on neuroimaging [47]. Follow-up MRI brain at 3-6 months is recommended to assess the evolution of ADEM lesions and verify whether they regress in a pattern commonly seen with ADEM. This study can also serve as a baseline for comparison for possible future episodes of acute demyelination.

\section{Treatments for Relapsing Demyelination}

Long-term clinical and radiological follow-up of ADEM is important. Sometimes, another episode of ADEM can occur more than 3 months following the initial insult and is referred to as multiphasic ADEM [21]. In other cases, a demyelinating episode not meeting criteria for ADEM (i.e., optic neuritis, transverse myelitis, clinically isolated syndrome) can occur and should prompt the clinician to address the possibility of a relapsing CNS demyelinating disorder.

In particular, if MOG IgG or NMO/aquaporin-4 IgG are detected, a child is at risk for future attacks and may benefit from immunosuppressive or immunomodulatory medications. We suggest that any child with an acute demyelinating syndrome who meets diagnostic criteria for NMOSD should immediately be started on a long-term immune therapy. Commonly used agents include rituximab and mycophenolate mofetil [48].

If a child develops ADEM as a part of MOG antibody disease, we suggest treating the acute attack, but not necessarily initiating immunosuppressive therapy unless a child has additional attacks. In our practice, we assess MOG antibodies at 6 and 12 months following the incident case of ADEM. If the child seroconverts to MOG IgG negative, further demyelinating events are unlikely. The persistence of MOG antibodies over time may confer a risk of future attacks, though the value of sequential testing and the relevance of the magnitude of its titer is controversial and warrants further study [49].

\section{Long-Term Follow-Up and Prognosis}

Monophasic ADEM is defined as no further disease activity as evidenced by no clinical attacks or radiological evidence of new CNS lesions 3 months after the initial episode. In monophasic ADEM, children commonly make a seemingly full recovery, usually over 4-6 weeks. A minority may have persistent cognitive and/or physical deficits, including difficulties with attention, slower processing speed, deficits in visual motor integration, and poor executive functioning [50-53]. We follow children every 6-12 months after ADEM to assess for emergence of cognitive or behavioral sequelae of the illness, such as specific learning disabilities, cognitive and physical fatigue, attention-deficit/hyperactivity disorder, mood disorders, and seizures, among others. A comprehensive neuropsychological evaluation when there is concern for these issues can be helpful in guiding parental expectations and facilitating appropriate school accommodations.

There has not been sufficient research on the effect of early versus delayed implementation of acute therapies and how aggressively second-line therapies should be pursued in an effort to optimize long-term neurocognitive outcomes in pediatric ADEM. Research from autoimmune encephalitis suggests that early initiation of immunotherapies and escalation to second-line therapies when there is poor response to first-line therapies is associated with better prognosis [54]. However, our center and others has found significant differences in autoimmune encephalitis and demyelinating CNS disorders [55], so how to best initiate and sequence acute treatments remains unknown.

\section{Conclusions}

ADEM is an acute demyelinating syndrome of the CNS, likely of post-infectious etiology. It can have many different presenting features, but the hallmark is multifocal neurological deficits associated with encephalopathy. Diagnostic workup involves serum and spinal fluid studies to investigate infectious and inflammatory causes and neuroimaging to characterize the extent of CNS involvement. Acute therapies are aimed at reducing inflammation and generally lead to marked clinical improvement. Studies obtained at presentation can help provide prognostic information as relates to relapsing CNS demyelinating syndromes. Long-term follow-up is essential to mitigate any potential neurological or psychosocial sequelae of the 


\section{Treatment Algorithm in ADEM}

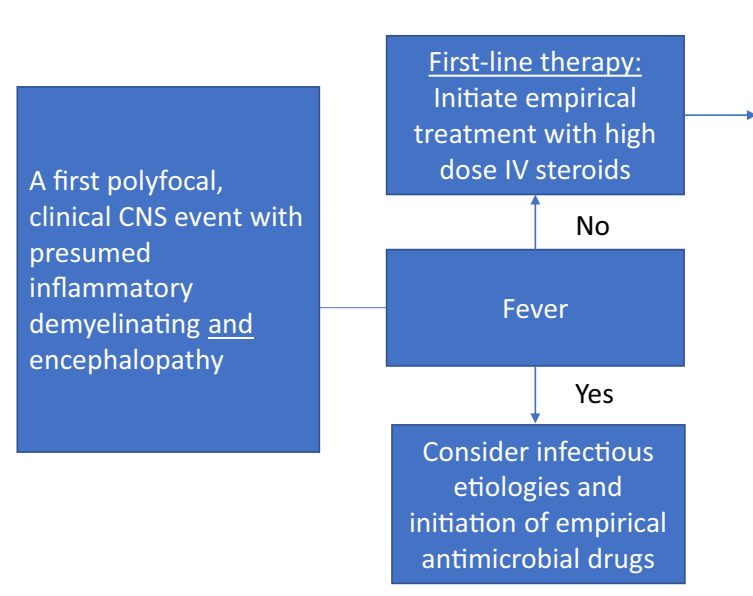

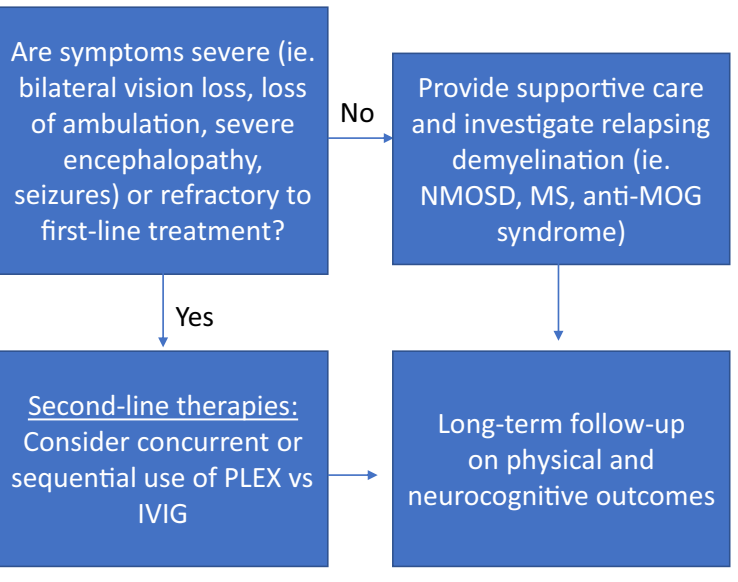

Fig. 1 Treatment algorithm for acute disseminated encephalomyelitis. $C N S$ central nervous system, IV intravenous, IVIG intravenous immunoglobulin, $M O G$ myelin oligodendrocyte glycoprotein, $M S$ multiple

condition. Figure 1 describes the treatment algorithm for ADEM in the pediatric patient.

\section{Declarations}

Funding No sources of funding were used to conduct this study or prepare this manuscript.

Conflict of interest Cynthia X. Wang has no conflicts of interest that are directly relevant to the content of this article.

Ethics approval Not applicable.

Consent to participate Not applicable.

Consent for publication Not applicable.

Availability of data and material Not applicable.

Code availability Not applicable.

Authors' contributions The author has written, read, and approved the final manuscript.

\section{References}

1. Stuve O, Zamvil SS. Pathogenesis, diagnosis, and treatment of acute disseminated encephalomyelitis. Curr Opin Neurol. 1999;12:395-401. https://doi.org/10.1097/00019052-19990 8000-00005.

2. Albert LJ, Inman RD. Molecular mimicry and autoimmunity. $\mathrm{N}$ Engl J Med. 1999;341:2068-74. https://doi.org/10.1056/NEJM1 99912303412707.

3. Pohl-Koppe A, Burchett SK, Thiele EA, et al. Myelin basic protein reactive $\mathrm{Th} 2 \mathrm{~T}$ cells are found in acute disseminated sclerosis, NMOSD neuromyelitis optica spectrum disorder, PLEX therapeutic plasma exchange

encephalomyelitis. J Neuroimmunol. 1998;91:19-27. https://doi. org/10.1016/s0165-5728(98)00125-8.

4. Tenembaum S, Chitnis T, Ness J, et al. Acute disseminated encephalomyelitis. Neurology. 2007;68:S23-36. https://doi.org/ 10.1212/01.wnl.0000259404.51352.7f.

5. Parsons T, Banks S, Bae C, et al. COVID-19-associated acute disseminated encephalomyelitis (ADEM). J Neurol. 2020. https:// doi.org/10.1007/s00415-020-09951-9.

6. Paterson RW, Brown RL, Benjamin L, et al. The emerging spectrum of COVID-19 neurology: clinical, radiological and laboratory findings. Brain. 2020. https://doi.org/10.1093/brain/awaa240.

7. Jorens PG, VanderBorght A, Ceulemans B, et al. Encephalomyelitis-associated antimyelin autoreactivity induced by streptococcal exotoxins. Neurology. 2000;54:1433-41. https://doi.org/10.1212/ wnl.54.7.1433.

8. Murthy JM. MRI in acute disseminated encephalomyelitis following Semple antirabies vaccine. Neuroradiology. 1998;40:420-3. https://doi.org/10.1007/s002340050615.

9. Baxter R, Lewis E, Goddard K, et al. Acute demyelinating events following vaccines: a case-centered analysis. Clin Infect Dis. 2016;63:1456-62. https://doi.org/10.1093/cid/ciw607.

10. Huynh W, Cordato DJ, Kehdi E, et al. Post-vaccination encephalomyelitis: literature review and illustrative case. J Clin Neurosci. 2008;15:1315-22. https://doi.org/10.1016/j.jocn.2008.05.002.

11. Bennetto L, Scolding N. Inflammatory/post-infectious encephalomyelitis. J Neurol Neurosurg Psychiatry. 2004;75(1):i22-28.

12. Banwell B, Kennedy J, Sadovnick D, et al. Incidence of acquired demyelination of the CNS in Canadian children. Neurology. 2009;72:232-9. https://doi.org/10.1212/01.wnl.0000339482. 84392.bd.

13. Leake JA, Albani S, Kao AS, et al. Acute disseminated encephalomyelitis in childhood: epidemiologic, clinical and laboratory features. Pediatr Infect Dis J. 2004;23:756-64. https://doi.org/10. 1097/01.inf.0000133048.75452.dd.

14. Cole J, Evans E, Mwangi M, et al. Acute disseminated encephalomyelitis in children: an updated review based on current diagnostic criteria. Pediatr Neurol. 2019;100:26-34. https://doi.org/ 10.1016/j.pediatrneurol.2019.06.017. 
15. Langer-Gould A, Zhang JL, Chung J, et al. Incidence of acquired CNS demyelinating syndromes in a multiethnic cohort of children. Neurology. 2011;77:1143-8. https://doi.org/10.1212/WNL.0b013 e31822facdd.

16. Pavone P, Pettoello-Mantovano M, Le Pira A, et al. Acute disseminated encephalomyelitis: a long-term prospective study and meta-analysis. Neuropediatrics. 2010;41:246-55. https://doi.org/ 10.1055/s-0031-1271656.

17. Absoud M, Lim MJ, Chong WK, et al. Paediatric acquired demyelinating syndromes: incidence, clinical and magnetic resonance imaging features. Mult Scler. 2013;19:76-86. https://doi.org/10. $1177 / 1352458512445944$.

18. Murthy JM. Acute disseminated encephalomyelitis. Neurol India. 2002;50:238-43.

19. Leake JA, Billman GF, Nespeca MP, et al. Pediatric acute hemorrhagic leukoencephalitis: report of a surviving patient and review. Clin Infect Dis. 2002;34:699-703. https://doi.org/10.1086/ 338718.

20. Singh RR, Sedani S, Lim M, et al. RANBP2 mutation and acute necrotizing encephalopathy: 2 cases and a literature review of the expanding clinico-radiological phenotype. Eur J Paediatr Neurol. 2015;19:106-13. https://doi.org/10.1016/j.ejpn.2014.11.010.

21. Krupp LB, Tardieu M, Amato MP, et al. International Pediatric Multiple Sclerosis Study Group criteria for pediatric multiple sclerosis and immune-mediated central nervous system demyelinating disorders: revisions to the 2007 definitions. Mult Scler. 2013;19:1261-7. https://doi.org/10.1177/1352458513484547.

22. Wang C, Narayan R, Greenberg B. Anti-myelin oligodendrocyte glycoprotein antibody associated with gray matter predominant transverse myelitis mimicking acute flaccid myelitis: a presentation of two cases. Pediatr Neurol. 2018;86:42-5. https://doi.org/ 10.1016/j.pediatrneurol.2018.06.003.

23. Dubey D, Pittock SJ, Krecke KN, et al. Clinical, radiologic, and prognostic features of myelitis associated with myelin oligodendrocyte glycoprotein autoantibody. JAMA Neurol. 2018. https:// doi.org/10.1001/jamaneurol.2018.4053.

24. Franciotta D, Columba-Cabezas S, Andreoni L, et al. Oligoclonal $\mathrm{IgG}$ band patterns in inflammatory demyelinating human and mouse diseases. J Neuroimmunol. 2008;200:125-8. https://doi. org/10.1016/j.jneuroim.2008.06.004.

25. Oliveira LM, Apostolos-Pereira SL, Pitombeira MS, et al. Persistent MOG-IgG positivity is a predictor of recurrence in MOGIgG-associated optic neuritis, encephalitis and myelitis. Mult Scler. 2019;25:1907-14. https://doi.org/10.1177/1352458518 811597

26. Wilson MR, Sample HA, Zorn KC, et al. Clinical metagenomic sequencing for diagnosis of meningitis and encephalitis. N Engl J Med. 2019;380:2327-40. https://doi.org/10.1056/NEJMoa1803 396.

27. Tenembaum S, Chamoles N, Fejerman N. Acute disseminated encephalomyelitis: a long-term follow-up study of 84 pediatric patients. Neurology. 2002;59:1224-31. https://doi.org/10.1212/ wnl.59.8.1224

28. Pohl D, Alper G, Van Haren K, et al. Acute disseminated encephalomyelitis: updates on an inflammatory CNS syndrome. Neurology. 2016;87:S38-45. https://doi.org/10.1212/WNL.0000000000 002825 .

29. Waldman AT, Gorman MP, Rensel MR, et al. Management of pediatric central nervous system demyelinating disorders: consensus of United States neurologists. J Child Neurol. 2011;26:67582. https://doi.org/10.1177/0883073810395141.

30. Salvador E, Shityakov S, Forster C. Glucocorticoids and endothelial cell barrier function. Cell Tissue Res. 2014;355:597-605. https://doi.org/10.1007/s00441-013-1762-z.
31. Coutinho AE, Chapman KE. The anti-inflammatory and immunosuppressive effects of glucocorticoids, recent developments and mechanistic insights. Mol Cell Endocrinol. 2011;335:2-13. https://doi.org/10.1016/j.mce.2010.04.005.

32. Dale RC, de Sousa C, Chong WK, et al. Acute disseminated encephalomyelitis, multiphasic disseminated encephalomyelitis and multiple sclerosis in children. Brain. 2000;123(12):2407-22. https://doi.org/10.1093/brain/123.12.2407.

33. Anlar B, Basaran C, Kose G, et al. Acute disseminated encephalomyelitis in children: outcome and prognosis. Neuropediatrics. 2003;34:194-9. https://doi.org/10.1055/s-2003-42208.

34. Borras-Novell C, Garcia Rey E, Perez Baena LF, et al. Therapeutic plasma exchange in acute disseminated encephalomyelitis in children. J Clin Apher. 2015;30:335-9. https://doi.org/10.1002/ jca. 21388 .

35. Manguinao M, Krysko KM, Maddike S, et al. A retrospective cohort study of plasma exchange in central nervous system demyelinating events in children. Mult Scler Relat Disord. 2019;35:50 4. https://doi.org/10.1016/j.msard.2019.07.004.

36. Pradhan S, Gupta RP, Shashank S, et al. Intravenous immunoglobulin therapy in acute disseminated encephalomyelitis. J Neurol Sci. 1999;165:56-61. https://doi.org/10.1016/s0022-510x(99) 00072-6.

37. Nishikawa M, Ichiyama T, Hayashi T, et al. Intravenous immunoglobulin therapy in acute disseminated encephalomyelitis. Pediatr Neurol. 1999;21:583-6. https://doi.org/10.1016/s0887-8994(99) 00042-9.

38. Sahlas DJ, Miller SP, Guerin M, et al. Treatment of acute disseminated encephalomyelitis with intravenous immunoglobulin. Neurology. 2000;54:1370-2. https://doi.org/10.1212/wnl.54.6. 1370.

39. Narayan RN, Wang C, Greenberg BM. Acute disseminated encephalomyelitis (ADEM) and increased intracranial pressure associated with anti-myelin oligodendrocyte glycoprotein antibodies. Pediatr Neurol. 2019;99:64-8. https://doi.org/10.1016/j.pedia trneurol.2019.03.009.

40. Orbach R, Schneebaum Sender N, Lubetzky R, et al. Increased intracranial pressure in acute disseminated encephalomyelitis. J Child Neurol. 2019;34:99-103. https://doi.org/10.1177/08830 73818811541.

41. Rodriguez-Porcel F, Hornik A, Rosenblum J, et al. Refractory fulminant acute disseminated encephalomyelitis (ADEM) in an adult. Front Neurol. 2014;5:270. https://doi.org/10.3389/fneur. 2014.00270

42. Ayed H, Chaudhary MW, AlBaradie R, et al. Use of cyclophosphamide in a child with fulminant acute disseminated encephalomyelitis. Child Neurol Open. 2018;5:2329048X18754631. https:// doi.org/10.1177/2329048X18754631.

43. Jaskowiak JM. Treatment of hemorrhagic acute disseminated encephalomyelitis with cyclophosphamide. Am J Health Syst Pharm. 2016;73:202-5. https://doi.org/10.2146/ajhp150079.

44. Makuuchi Y, Nishimoto M, Yamamoto K, et al. Successful treatment with rituximab in acute disseminated encephalomyelitis with whole spinal cord involvement following HLA haploidentical transplantation. Rinsho Ketsueki. 2018;59:2588-93. https:// doi.org/10.11406/rinketsu.59.2588.

45. Alexander M, Murthy JM. Acute disseminated encephalomyelitis: Treatment guidelines. Ann Indian Acad Neurol. 2011;14:S60-64. https://doi.org/10.4103/0972-2327.83095.

46. Carlisi E, Pavese C, Mandrini S, et al. Early rehabilitative treatment for pediatric acute disseminated encephalomyelitis: case report. Eur J Phys Rehabil Med. 2015;51:341-3.

47. O'Riordan JI, Gomez-Anson B, Moseley IF, et al. Long term MRI follow-up of patients with post infectious encephalomyelitis: 
evidence for a monophasic disease. J Neurol Sci. 1999;167:132-6. https://doi.org/10.1016/s0022-510x(99)00160-4.

48. Gombolay GY, Chitnis T. Pediatric neuromyelitis optica spectrum disorders. Curr Treat Options Neurol. 2018;20:19. https://doi.org/ 10.1007/s11940-018-0502-9.

49. Hacohen Y, Banwell B. Treatment approaches for MOG-Abassociated demyelination in children. Curr Treat Options Neurol. 2019;21:2. https://doi.org/10.1007/s11940-019-0541-x.

50. Beatty C, Bowler RA, Farooq O, et al. Long-term neurocognitive, psychosocial, and magnetic resonance imaging outcomes in pediatric-onset acute disseminated encephalomyelitis. Pediatr Neurol. 2016;57:64-73. https://doi.org/10.1016/j.pediatrneurol. 2016.01.003.

51. Shilo S, Michaeli O, Shahar E, et al. Long-term motor, cognitive and behavioral outcome of acute disseminated encephalomyelitis. Eur J Paediatr Neurol. 2016;20:361-7. https://doi.org/10.1016/j. ejpn.2016.01.008.
52. Hahn CD, Miles BS, MacGregor DL, et al. Neurocognitive outcome after acute disseminated encephalomyelitis. Pediatr Neurol. 2003;29:117-23. https://doi.org/10.1016/s0887-8994(03)00143-7.

53. Deery B, Anderson V, Jacobs R, et al. Childhood MS and ADEM: investigation and comparison of neurocognitive features in children. Dev Neuropsychol. 2010;35:506-21. https://doi.org/10. 1080/87565641.2010.494921.

54. Titulaer MJ, McCracken L, Gabilondo I, et al. Treatment and prognostic factors for long-term outcome in patients with antiNMDA receptor encephalitis: an observational cohort study. Lancet Neurol. 2013;12:157-65. https://doi.org/10.1016/S14744422(12)70310-1.

55. McGetrick ME, Varughese NA, Miles DK, et al. Clinical features, treatment strategies, and outcomes in hospitalized children with immune-mediated encephalopathies. Pediatr Neurol. 2021;116:20-6. https://doi.org/10.1016/j.pediatrneurol.2020.11. 014. 